\title{
Front Matter: Volume 9892
}

, "Front Matter: Volume 9892," Proc. SPIE 9892, Semiconductor Lasers and Laser Dynamics VII, 989201 (2 August 2016); doi: 10.1117/12.2243911

SPIE. Event: SPIE Photonics Europe, 2016, Brussels, Belgium 


\title{
PROCEEDINGS OF SPIE
}

\section{Semiconductor Lasers \\ and Laser Dynamics VII}

\author{
Krassimir Panajotov \\ Marc Sciamanna \\ Angel Valle \\ Rainer Michalzik \\ Editors
}

\author{
4-7 April 2016 \\ Brussels, Belgium \\ Sponsored by \\ SPIE \\ Cosponsored by \\ B-PHOT-Brussels Photonics Team (Belgium) \\ Research Foundation Flanders (Belgium) \\ Visit Brussels (Belgium) \\ Cooperating Organisations \\ Photonics 21 (Germany) \\ EOS-European Optical Society (Germany) \\ KTN-the Knowledge Transfer Network (United Kingdom) \\ Graphene Flagship (Belgium) \\ Photonics Public Private Partnership (Belgium) \\ Published by \\ SPIE
}

Volume 9892

Proceedings of SPIE 0277-786X, V. 9892

SPIE is an international society advancing an interdisciplinary approach to the science and application of light.

Semiconductor Lasers and Laser Dynamics VII, edited by Krassimir Panajotov, Marc Sciamanna,

Angel Valle, Rainer Michalzik, Proc. of SPIE Vol. 9892, 989201 · (C) 2016 SPIE

CCC code: $0277-786 \mathrm{X} / 16 / \$ 18 \cdot$ doi: $10.1117 / 12.2243911$

Proc. of SPIE Vol. $9892989201-1$ 
The papers in this volume were part of the technical conference cited on the cover and title page. Papers were selected and subject to review by the editors and conference program committee. Some conference presentations may not be available for publication. Additional papers and presentation recordings may be available online in the SPIE Digital Library at SPIEDigitallibrary.org.

The papers reflect the work and thoughts of the authors and are published herein as submitted. The publisher is not responsible for the validity of the information or for any outcomes resulting from reliance thereon.

Please use the following format to cite material from these proceedings:

Author(s), "Title of Paper," in Semiconductor Lasers and Laser Dynamics VII, edited by Krassimir Panajotov, Marc Sciamanna, Angel Valle, Rainer Michalzik, Proceedings of SPIE Vol. 9892 (SPIE, Bellingham, WA, 2016) Six-digit Article CID Number.

ISSN: 0277-786X

ISSN: 1996-756X (electronic)

ISBN: 9781510601376

Published by

SPIE

P.O. Box 10, Bellingham, Washington 98227-0010 USA

Telephone +1 3606763290 (Pacific Time) · Fax +1 3606471445

SPIE.org

Copyright (C) 2016, Society of Photo-Optical Instrumentation Engineers.

Copying of material in this book for internal or personal use, or for the internal or personal use of specific clients, beyond the fair use provisions granted by the U.S. Copyright Law is authorized by SPIE subject to payment of copying fees. The Transactional Reporting Service base fee for this volume is $\$ 18.00$ per article (or portion thereof), which should be paid directly to the Copyright Clearance Center (CCC), 222 Rosewood Drive, Danvers, MA 01923. Payment may also be made electronically through CCC Online at copyright.com. Other copying for republication, resale, advertising or promotion, or any form of systematic or multiple reproduction of any material in this book is prohibited except with permission in writing from the publisher. The CCC fee code is 0277-786X/16/\$18.00.

Printed in the United States of America.

Publication of record for individual papers is online in the SPIE Digital Library.

\section{SPIE. DIGITAL}

Paper Numbering: Proceedings of SPIE follow an e-First publication model. A unique citation identifier (CID) number is assigned to each article at the time of publication. Utilization of CIDs allows articles to be fully citable as soon as they are published online, and connects the same identifier to all online and print versions of the publication. SPIE uses a six-digit CID article numbering system structured as follows:

- The first four digits correspond to the SPIE volume number.

- The last two digits indicate publication order within the volume using a Base 36 numbering system employing both numerals and letters. These two-number sets start with 00, 01, 02, 03, 04, $05,06,07,08,09,0 A, 0 B \ldots$ OZ, followed by 10-1Z, 20-2Z, etc. The CID Number appears on each page of the manuscript. 


\title{
Contents
}

\author{
vii Authors \\ ix Conference Committee
}

\section{SESSION 1 PHOTONIC CRYSTAL LASERS}

989204 Lasing dynamics of photonic crystal reflector laser [9892-3]

\section{SESSION 2 VCSELS I}

989206 Birefringent vertical cavity surface-emitting lasers: toward high-speed spin-lasers (Invited Paper) [9892-5]

989208 VCSELs with optically controlled current confinement: experiments and analysis [9892-7]

\section{SESSION 3 QUANTUM DOT LASERS I}

9892 OA High-speed directly modulated $1.5 \mu \mathrm{m}$ quantum dot lasers (Invited Paper) [9892-9]

$98920 \mathrm{C}$ InAs/GaAs excited state quantum-dot transmitters operating under long-delayed optical feedback [9892-11]

\section{SESSION 4 LASER DYNAMICS I}

9892 OD Phase noise reduction in semiconductor lasers by optical negative feedback (Invited Paper) [9892-12]

9892 OE Effects of modulation in the complex dynamics of a semiconductor laser with feedback (Invited Paper) [9892-13]

9892 OG Improving the chaos bandwidth of a semiconductor laser with phase-conjugate feedback [9892-15]

$9892 \mathrm{OH}$ Sparse signal reconstruction based on experimental chaos generated by a laser diode [9892-16]

\section{SESSION 5 LASER DYNAMICS II}

98920 ol Delay differential models in multimode laser dynamics: taking chromatic dispersion into account (Invited Paper) [9892-17]

9892 OK Nonlinear dynamics in a semiconductor laser subject to filtered phase-conjugate optical feedback [9892-19] 
$9892 \mathrm{OL}$ Investigating optical complexity of the phase transition in the intensity of a fibre laser radiation [9892-20]

$98920 \mathrm{M}$ Complexity of chaos in three cascaded vertical-cavity surface-emitting lasers [9892-21]

\section{SESSION 6 VCSELS II}

9892 OP Investigating the threshold properties of very small VCSELs through relaxation-resonanceinduced amplification [9892-24]

$98920 Q$ Theoretical and experimental study of polarization switching in long-wavelength VCSELs subject to parallel optical injection [9892-25]

9892 OR Parameter extraction from temperature-dependent light-current-voltage data of verticalcavity surface-emitting lasers [9892-26]

\section{SESSION 7 QUANTUM DOT LASERS II}

98920 QS Quantum-dot lasers for $35 \mathrm{Gbit} / \mathrm{s}$ pulse-amplitude modulation and $160 \mathrm{Gbit} / \mathrm{s}$ differential quadrature phase-shift keying (Invited Paper) [9892-27]

9892 OT Optical nonlinearities in InAs/GaAs injection-locked quantum dot light-based emitters (Invited Paper) [9892-28]

$98920 \mathrm{U}$ Investigations on the relative intensity noise of a two-state quantum dot laser [9892-29]

$98920 \mathrm{~V}$ Dual state antiphase excitability in optically injected quantum dot lasers [9892-30]

9892 OW Range-dependent effects of optical feedback on multimode two-color quantum dot lasers [9892-31]

\section{SESSION 8 GAN LASERS}

9892 OY Progress and challenges in electrically pumped GaN-based VCSELs (Invited Paper) [9892-33]

$98920 Z$ AIGalnN laser diode bar and array technology for high-power and individual addressable applications [9892-34]

\section{SESSION 9 VECSELS AND HIGH-POWER LASERS}

989211 Capacitance and modulation time constant in oxide-confined vertical-cavity surfaceemitting lasers with different oxide layers (Invited Paper) [9892-36] 
989214 Capsule-shaped metallic-cavity semiconductor lasers for low-energy on-chip light sources (Invited Paper) [9892-39]

989216 Effects of optical backscattering on silicon photonic hybrid laser performance [9892-41]

989217 Low-linewidth and tunable single frequency $1 \times 2$ multimode-interferometer-Fabry-Perot laser [9892-42]

\section{SESSION 11 CASCADE LASERS AND NEW EDGE EMITTERS}

$98921 \mathrm{~A}$ Recent progress on intensity and chirp compensation of EADFB laser realized by SOA integration (Invited Paper) [9892-44]

9892 1B Red to green emitters from InGaP/InAlGaP laser structure by strain-induced quantum-well intermixing [9892-46]

\section{SESSION 12 CAVITY SOLITONS}

9892 1E Polarization properties of localized structures in VCSELs (Best Student Paper Award) [9892-49]

9892 IF Weakly nonlinear analysis and localised structures in nonlinear cavities with metamaterials [9892-50]

\section{SESSION 13 LASERS WITH DELAYED OPTICAL FEEDBACK AND RING LASERS}

9892 1G Delayed feedback control of cavity solitons in a broad area vertical cavity surface emitting laser with saturable absorbtion (Invited Paper) [9892-51]

$98921 \mathrm{~J}$ Dynamics of semiconductor microring lasers subject to on-chip filtered optical feedback [9892-55]

POSTER SESSION

9892 IL Analysis of the light-field intensity dependence of catastrophic optical damage in highpower AIGalnP lasers using an asymmetrical tapered laser [9892-56]

$98921 \mathrm{M}$ Chaos in solitary VCSELs: asymmetry and noise [9892-57]

9892 iN One way synchronization of polarization chaos from a solitary Vertical-Cavity SurfaceEmitting Laser [9892-58]

989210 Recurrent state-switching of a two-state quantum dot laser by optical feedback [9892-59] 
9892 IP A new technique for ultrafast physical random number generation using optical chaos [9892-60]

9892 iR Self-aligned BCB planarization method for high-frequency signal injection in a VCSEL with an integrated modulator [9892-63]

9892 is Chaos synchronization in mutually coupled long-wavelength vertical-cavity surfaceemitting lasers with long delay time [9892-64]

$98921 \mathrm{~T} \quad$ Analysis of the effects of periodic forcing in the spike rate and spike correlation's in semiconductor lasers with optical feedback [9892-65]

9892 IV Optimal parameters of monolithic high-index contrast grating VCSELs [9892-67]

$98921 Y$ Analysis of optical responses of $1060 \mathrm{~nm}$ seed laser diodes under overcurrent and shortpulse conditions for reliability investigations [9892-70]

$98921 Z$ Reading a CD-ROM without a photodiode [9892-71]

989220 Dynamics of quantum cascade lasers: numerics [9892-72]

$989221 \quad$ Mixed transverse modes in coupled-cavity VCSELs [9892-73]

989222 Birefringence tuning of VCSELs [9892-74]

989224 Frequency tuning of polarization oscillations in spin-polarized vertical-cavity surfaceemitting lasers [9892-76]

989225 Amplitude jitter and timing jitter characterization of a monolithic high-power passively mode-locked tapered quantum dot laser [9892-77]

989227 Self-organized light bullets in type-I intracavity second-harmonic generation [9892-79]

989228 Stabilization of spatio-temporal instabilities in optically injected broad-area lasers [9892-80]

989229 Temperature-induced laser dynamics in wide-aperture VCSELs [9892-81] 


\section{Authors}

Numbers in the index correspond to the last two digits of the six-digit citation identifier (CID) article numbering system used in Proceedings of SPIE. The first four digits reflect the volume number. Base 36 numbering is employed for the last two digits and indicates the order of articles within the volume. Numbers start with 00, 01, 02, 03, 04, 05, 06, 07, 08, 09, OA, OB...0Z, followed by 10-1Z, 20-2Z, etc.

Abdollahinia, Alireza, OA Ackemann, Thorsten, $1 \mathrm{E}$ Al-Jabr, A. A., 1B Almuneau, Guilhem, $1 R$ Anchikov, D. A., 28, 29

Aoyama, Konosuke, OD Aragoneses, Andrés, $\mathrm{OE}, \mathrm{OL}$ Arsenijević, Dejan, OC, OS, OT Averlant, Etienne, 1E, 27 Bader, Sven, 08

Bakoz, Andrei P., 04 Banyoudeh, Saddam, OA

Béchou, L., IY

Bengtsson, J., OY Bimberg, Dieter, OC, OS, OT Blaha, S., 27 Boćkowski, M., $\mathrm{OZ}$

Bou Sanayeh, Marwan, 1L, 22 Brever, Stefan, OU, OW, 10, 25

Calciati, M., OY

Carlsson, S., OY

Carpi, Laura, OL

Chang, C.-Y., $\mathrm{OH}$

Choi, D., $\mathrm{OH}$

Choquette, Kent, 21

Churkin, Dmitri V., OL

Citrin, D. S., $\mathrm{OH}, 1 \mathrm{Z}$

Corbett, Brian, 17

Czernecki, R., $0 Z$

Czyszanowski, Tomasz, 1V, 21

Danckaert, Jan, $1 \mathrm{~J}$

Daubenschüz, Markus, OR

Dems, Maciej, $1 \mathrm{~V}$

Doucet, Jean-Baptiste, $1 R$

Drzewietzki, Lukas, 25

Dubinkin, I., OV

Eisenstein, Gadi, OA

Elsäßer, Wolfgang, OU, OW

Elsonbaty, Amr, IP

Erneux, Thomas, OK, OV, $1 \mathrm{~J}$

Eyal, Ori, OA

Fedorov, N., OV

Frasunkiewicz, Leszek, 21

Friart, Gaetan, 1J

Fujiwara, N., 1A

Gebski, Marcin, 1V

Gerhardt, Nils C., 06, 22, 24

Gerlach, Philipp, 08

Gioannini, M., OU
Goano, M., OY

Goulding, David, OV

Grillot, Frédéric, OC, OT

Gustavsson, J., OY

Gurevich, S. V., $1 G$

Haglund, ^̊, OY

Habruseva, T., 04

Hasebe, K., 1 A

Hashemi, E., OY

Hegarty, Stephen P., 04, OV

Hegazy, Salem F., IP

Hofmann, Martin R., 06, 22, 24

Hong, Yanhua, OM, OQ

Huang, Heming, OC, OT

Huyet, Guillaume, 04, Ol, OV

Itoh, M., IA

Joly, S., $1 Y$

Kanazawa, S., IA

Kelleher, Bryan, OV

Khoder, Mulham, $1 \mathrm{~J}$

Klein, J., 16

Kobayashi, W., IA

Krents, A. A., 28, 29

Krestin, S. V., 29

Kucharski, R., $\mathrm{OZ}$

Laruelle, F., $1 Y$

Le Galès, G., $1 Y$

Lecestre, Aurélie, 1R

Leszczyński, M., OZ

Liles, A. A., 04

Lindemann, Markus, 06, 22, 24

Lippi, G. L., OP

Locquet, Alexandre, $\mathrm{OH}, 1 \mathrm{Z}$

Lott, James A., 11

Majid, M. A., 1B

Makhoute, A., IF

Marciniak, Magdalena, IV

Marigo-Lombart, Ludovic, IR

Marona, L., OZ

Masoller, Cristina, OE, OL, IT

Mercier, Émeric, OG

Michalzik, Rainer, 06, 08, 0R, 22, 24

Molevich, N. E., 28, 29

Morisset, A., $1 Y$

Morrissey, Padraic, 17

Moser, Philip, 11

Mourozeau, G., 1 Z

Najda, S. P., $0 Z$

Nakano, Yoshiaki, 14 
$\mathrm{Ng}, \mathrm{K} ., 1 \mathrm{Z}$

$\mathrm{Ng}, \mathrm{T} . \mathrm{K} ., 1 \mathrm{~B}$

Obayya, Salah S. A., IP

O'Faolain, L., 04

Ooi, B. S., 1B

O'Shaughnessy, Ben,

Pacradouni, V., 16

Pakhomov, A. V., 28, 29

Panajotov, Krassimir, OM, 0Q, OW, 1E, 1G, 1M, 1N,

$10,1 R, 1 S, 21,27$

Pawlus, Robert, OU, OW

Pedroza, G., $1 Y$

Pérez, $P ., O Q$

Perlin, $\mathrm{P} ., \mathrm{OZ}$

Perrone, Sandro, OE

Pesquera, L., OQ

Peters, Frank H., 17

Pimenov, A., Ol

Pond, J., 16

Popp, A., OQ

Puccioni, G. P., OP

Pusch, Tobias, 06, 22, 24

Quintero, Carlos A., OE

Quintero-Quiroz, C., $1 T$

Quirce, Ana, OM, 0Q, 1S

Reig, Benjamin, $1 R$

Reithmaier, Johann Peter, OA

Rontani, D., $\mathrm{OH}$

Rousset, Bernard, 1R

Sadeev, Tagir, 0C, OT

Sahai, A. A., 1 Z

Sanjoh, H., $1 \mathrm{~A}$

Schelte, C., IG

Schires, Kevin, OC, OT

Schnabel, Florian, OA

Sciamanna, Marc, 0G, OK, OW, 1M, 1N, 10

Sichkovskyi, Vitalii, OA

Shen, C., 1B

Slimani, N., 1F

Sorrentino, Taciano, OE, $1 T$

Spencer, Paul S., OM

Śpiewak, Patrycja, 11

Stattin, M., OY

Suski, T., OZ

Tanemura, Takuo, 14

Tarasov, Nikita, OL

Targowski, G., $\mathrm{OZ}$

Thienpont, Hugo, OQ, 1E, 1M, 1N, 1R, IS

Tlidi, Mustapha, 1E, 1F, 1G, 27

Torrent, M. C., OE, OL, IT

Turitsyn, Sergei K., OL

Tykalewicz, B., OV

Valle, A., OQ, 1S

Van der Sande, Guy, 1J, 20

Verschaffelt, Guy, 1J, 20

Viktorov, E. A., 04, OV

Virte, Martin, OU, OW, 1M, 1N, 10

Vladimirov, A. G., Ol

Wang, Bingjie, $O M$

Wang, T., OP
Wasiak, Michał, 11

Weber, Christoph, 25

Weicker, Lionel, OK

Wishon, Michael J., 1 Z

Wisniewski, P., OZ

Wolfersberger, Delphine, OG, OK Yang, Hua, 17

Yang, Mingqi, 17

Yasaka, Hiroshi, OD

Yokota, Nobuhide, OD

Zhang, Baifu, 14 


\section{Conference Committee}

Symposium Chairs

Francis Berghmans, Vrije Universiteit Brussel (Belgium)

Jürgen Popp, Institut für Photonische Technologien e.V. (Germany)

Ronan Burgess, European Commission Photonics Unit (Belgium)

Peter Hartmann, SCHOTT AG (Germany)

Honorary Symposium Chair

Hugo Thienpont, Vrije Universiteit Brussel (Belgium)

Conference Chairs

Krassimir Panajotov, Vrije Universiteit Brussel (Belgium)

Marc Sciamanna, CentraleSupélec (France)

Angel Valle, Universidade de Cantabria (Spain)

Rainer Michalzik, Universität Ulm (Germany)

Conference Program Committee

Dieter Bimberg, Technische Universität Berlin (Germany)

Weng W. Chow, Sandia National Labs (United States)

Kent D. Choquette, University of Illinois at Urbana-Champaign (United States)

Gadi Eisenstein, Technion-Israel Institute of Technology (Israel)

Wolfgang E. Elsässer, Technische Universität Darmstadt (Germany)

Nicolas Grandjean, Ecole Polytechnique Fédérale de Lausanne (Switzerland)

Hitoshi Kawaguchi, Nara Institute of Science and Technology (Japan)

Norbert Lichtenstein, II-VI Laser Enterprise GmbH (Switzerland)

Cristina Masoller, Universidad Politècnica de Catalunya (Spain)

Jesper Mørk, Technical University of Denmark (Denmark)

Jerome V. Moloney, College of Optical Sciences, The University of

Arizona (United States)

Ivo Montrosset, Politecnico di Torino (Italy)

Wlodzimierz Nakwaski, Technical University of Lodz (Poland)

Gian-Luca Oppa, University of Strathclyde (United Kingdom)

Johann Peter Reithmaier, Universität Kassel (Germany)

Carlo Sirtori, Université Paris 7-Denis Diderot (France)

Session Chairs 
1 Photonic Crystal Lasers

Krassimir Panajotov, Vrije Universiteit Brussel (Belgium)

2 VCSELS I

Rainer Michalzik, Universität Ulm (Germany)

3 Quantum Dot Lasers I

Dejan Arsenijevic, Technische Universität Berlin (Germany)

4 Laser Dynamics I

Lionel Weicker, CentraleSupélec (France)

5 Laser Dynamics II

Angel Valle, Universidade de Cantabria (Spain)

6 VCSELS II

Fumio Koyama, Tokyo Institute of Technology (Japan)

7 Quantum Dot Lasers II

Sebastian Wieczorek, University College Cork (Ireland)

8 GaN Lasers

Rainer Michalzik, Universität Ulm (Germany)

9 VECSELs and High-Power Lasers

Tomasz G. Czyszanowski, Lodz University of Technology (Poland)

10 New Device Concepts

Angel Valle, Universidade de Cantabria (Spain)

11 Cascade Lasers and New Edge Emitters

Johann P. Reithmaier, Universität Kassel (Germany)

12 Cavity Solitons

Andrei G. Vladimirov, Weierstrass-Institut für Angewandte Analysis und Stochastik (Germany)

13 Lasers with Delayed Optical Feedback and Ring Lasers

Krassimir Panajutov, Vrije Universteit Brusels (Belgium) 